\title{
Small G Protein
}

National Cancer Institute

\section{Source}

National Cancer Institute. Small G Protein. NCI Thesaurus. Code C18277.

A class of small GT P-binding regulatory proteins regulated by G protein coupled receptors that act as switches in signal transduction pathways. The nature of the nucleotide bound to the protein determines its activity: GTP in the active state, GDP in the inactive state. The prototype for this class of proteins is the cellular proto-oncogene RAS. 